\title{
Dark molecular hydrogen
}

\section{Daniel Pfenniger*广}

Geneva Observatory, University of Geneva, Switzerland

E-mail: daniel.pfenniger@obs.unige.ch

We discuss work in progress about the existence and possible forms of molecular hydrogen as a dark matter component particularly in the outer galactic disks, and in late or low surface brightness galaxies. Mainly, the direct condensation of molecular hydrogen in solid or liquid form during gravitational collapses opens possibilities on the evolution of collapsing cold gas in the interstellar medium that differs from star or planet formation. Gas globules stabilized in their centre by a terrestrial planet-sized liquid or solid core of molecular hydrogen are possible long-lived structures that naturally explain a series of apparently distinct problems, such as the cometary globules in planetary nebulae and the relation of $\mathrm{HI}$ to dark matter in galactic disks.

Baryons in Dark Matter Halos

5-9 October 2004

Novigrad, Croatia

\footnotetext{
* Speaker.

${ }^{\dagger}$ This work has been supported by the Swiss National Science Foundation.
} 


\section{Baryonic Dark Matter in Spiral Galaxies}

In the recent years the evidences for substantially self-gravitating galactic outer disks have been reinforced. Not only new self-consistent Milky Way models taking into account numerous observational constraints favour a heavy disk (Kalberla, 2003), but also the spiral arm number for a known rotation curve in NGC 2915 provides another kind of constraint on the disk selfgravity (Masset \& Bureau, 2003). In addition, as presented elsewhere in this volume, the ubiquitous warps suggest also that galactic disks are more massive than the detected baryons by a factor several (Revaz \& Pfenniger, 2004) if they result from a gravitational bending instability. The advantage of this explanation is that the ultimate cause of spiral arms and warps is the same: a marginal gravitational stability state, radial and vertical respectively. Disk dark matter indicates a dissipational component. Since both baryonic and non-baryonic dark matters are expected to exist, we are now able to better characterize their respective amount and distribution in galaxies.

Cuillandre et al. (2001) and de Block \& Walter (2003) showed that star formation does occur in the extreme outer HI disks of M31 and NGC 6822, jointly to HI density and dust extinction. Although no $\mathrm{CO}$ emission has been detected, these young stars prove indirectly that a as yet undetected molecular gas component does exist. Indeed, to form stars the HI in outer galactic disks must fragment and become molecular first. This has obviously a direct consequence about the distribution and nature of dark matter in the outer regions of spirals, where the issue about flat rotation curves started. Allen (2004) proposes that the HI results from the photo-dissociated more massive $\mathrm{H}_{2}$ component, suggesting a very active outer galaxy ISM. From distinct observational considerations about dust emission in low metallicity and low excitation regions, Boulanger (2004) also concludes that much more $\mathrm{H}_{2}$ must be present in outer galactic disks. Significant amounts of dark cold HI in self-absorption has also been unveiled in high resolution surveys of the Galaxy neighbourhood without showing the expected CO counterpart (Gibson et al., 2000).

Another evidence that baryons play a larger role than previously admitted has been found while revisiting the work of McGaugh et al. (2000) about the Baryonic Tully-Fisher (TF) relation, where the detected baryons (stars and HI) were shown to better fit the TF relation than luminosity. With the help of advanced statistical tests (the Akaike and Schwartz tests) we could extend McGaugh et al. result by showing that the TF relation, using either the same galaxy sample or another independent sample, is significantly improved when the $\mathrm{HI}+\mathrm{He}$ mass is globally multiplied by a factor $\sim 3$ (Pfenniger \& Revaz, 2005). The same statistical tools allow to show that factors like a varying $M / L$ ratio or a quadratic term do not improve the TF relation significantly.

The arguments above favour more dark matter of baryonic kind in the disk, without eliminating the existence of non-baryonic dark matter at larger scales. It is therefore necessary to find models of baryonic dark matter that are both consistent with observations and physics, and distinct from the non-baryonic dark matter components relevant at cosmological scales.

The MACHO hypothesis having been largely falsified, the most promising dark baryon models are based on cold gas in various forms. The direct detection in CO with the IRAM interferometer of dense and fractal molecular clumps down to $300 \mathrm{AU}$ (Heithausen, 2004) brings a direct support to the models of dark molecular gas that we proposed earlier (Pfenniger \& Combes, 1994, hereafter PC94). This and other models of cold molecular gas had however stability problems that needed to be addressed. Below we summarize some results of an ongoing research. 


\section{Overview of a Model of Dark $\mathbf{H}_{2}$ Including Condensed Phases}

The earlier models of dark cold $\mathrm{H}_{2}$ could meet the observational constraints because most of the molecules of low temperature gas are in the ground state unable to radiate. Further, in such conditions and to be consistent with virial constraints, gas must be clumped so densely in AU sized structures that most of the mass covers only a small $(\sim 1 \%)$ fraction of the sky, making absorption studies still challenging today. Among other interesting properties, this form of dark baryons is still relatively weakly bound (of order of $0.001 \mathrm{eV} / \mathrm{H}$ ), which allows typical radiation fields in the optical disks of galaxies to evaporate such clumps within Gyr time-scales. Therefore, such clouds can play the role of a gas reservoir similar to HI giant clouds. AU-sized cold molecular clouds can also be optically thick to cosmic and $\gamma$ rays, which means that the cosmic rays do not probe necessarily all the baryons that can be easily released into the diffuse ISM since, as stars and planets, dense cold gas can be shielded from cosmic rays.

While the PC94 model focused on the observed fractal state of the cold molecular ISM, extrapolated to $10-100 \mathrm{AU}$ scales, the alternative models (e.g., Gerhard \& Silk, 1996) focused on stable gaseous spherical clouds of cosmological origin. Today the fractal model seems difficult to maintain over several Gyrs (molecular clouds are transient), but the same objection can be made for unconfined isothermal gas clouds, for which the dissolution time is much shorter than a Gyr. However, in PC94 we pointed out in such clouds $\mathrm{H}_{2}$ was close to condense in solid or liquid form, a state of hydrogen discussed only a few times since the 60's. For example Wardle \& Walker (1999) examined models of gas clouds in which $\mathrm{H}_{2}$ forms ice grains or liquid droplets from the gas phase.

Currently we examine the direct $\mathrm{H}_{2}$ gas-solid or gas-liquid conversion during gravitational collapses. With the help of simple models and particle simulations we have estimated what happens to a cold (below $33 \mathrm{~K}$ ) and low opacity $\mathrm{He}$ and $\mathrm{H}_{2}$ gas during a gravitational collapse. As well established, perfect gas collapses proceed almost isothermally as long as the central opacity is low enough for cooling to be faster than compression warming (Larson, 1969). The crucial point is: if a phase transition to a condensed form occurs before the core becomes opaque to its own thermal radiation, then the condensed core cools on compression instead of warming up. From the thermodynamical properties of $\mathrm{H}_{2}$ and $\mathrm{He}$ as well as the opacity of the ISM in the sub-mm wavelengths found in the literature, it turns out that the compression of $\mathrm{H}_{2}$ in sufficiently cold conditions (especially below $\sim 5 \mathrm{~K}$ ) allows to reach easily a condensed $\mathrm{H}_{2}$ phase in the core of a collapsing gas cloud. Solid form of $\mathrm{H}_{2}$ occurs below $13.8 \mathrm{~K}$, and liquid form below $33 \mathrm{~K}$.

The predicted equilibrium bodies consist of a condensed $\mathrm{H}_{2}$ core of typically terrestrial mass and size (with a density of about $0.1 \mathrm{~g} \mathrm{~cm}^{-3}$ ) surrounded by a quasi isothermal $\mathrm{He}+\mathrm{H}_{2}$ atmosphere that can be more massive and extended than the core. The globules are then more stable than pure isothermal clouds because their incompressible core prevents gravothermal instabilities. The main evolutive factors are heating sources such as cosmic rays and UV radiation, as well as a residual radioactivity. Due to the negative specific heat of such globules, when cosmic rays or radiation penetrate deep inside the atmosphere, the globules cool while expanding, reinforcing the condensation state of the core! Therefore the FIR response of such globules to ambient excitation must differ from the diffuse ISM one as discussed in Reach et al. (1995).

The described condensation process is rather fast $\left(\sim 10^{3}-10^{4} \mathrm{yr}\right)$, and requires mainly a low

opacity gas to the sub-mm radiation and a low temperature. Therefore, the process is expected 
to be ubiquitous in cold, low metallicity outer parts of galaxies. The derived globules properties resemble much the ones of cometary globules observed in nearby planetary nebulae, for which no satisfying models exist yet. The $\mathrm{H}_{2}$ globule lifetime should depend mostly on the ambient level of excitation, the condensed cores surviving the longest, up to several Gyr, in low excitation regions.

Thus outer galactic disks would be mostly composed of such globules or naked condensed cores. A minor fraction of the baryons would spend about 5-20\% of the time in the HI diffuse form, as a whole the "gas" would behave as an almost collisionless component. A small fraction of the collapses would lead to usual stars, whenever the temperature, or the opacity are too high for the core to meet the $\mathrm{H}_{2}$ condensation curve. Conversely, in the optical disks stars would form in a larger fraction. Star formation appears then as a self-encouraging process for the supplementary reason that stars raise the temperature and opacity of the surrounding gas, making it less likely for collapsing cold cores to meet the $\mathrm{H}_{2}$ gas-solid or gas-liquid phase transition.

In conclusion, the cold globules provide a same cause for disparate facts, among which: 1) the almost collisionless dynamics of disks, 2) Bosma's HI-dark matter trend, 3) the anti-correlation of dark matter and stars. Overall, isolated disk galaxies would mainly evolve by transforming insideout the dark gas into stars, starting from pure dark gas disks possibly embedded in a non-baryonic dark halo, to a short BCD phase with few stars, some HI, and much dark matter. Then along the spiral sequence from late to early types, galaxies would increasingly be dominated by stars and the residual non-baryonic dark matter.

\section{References}

Allen, R.J. 2004, in Penetrating Bars through Masks of Cosmic Dust, D. Block et al. (eds.), Kluwer, 731

Boulanger, F. 2004, in Penetrating Bars through Masks of Cosmic Dust, D. Block et al. (eds.), Kluwer, 749

Cuillandre, J.-C., Lequeux, J., Allen, R.J., Mellier, \& Y., Bertin, E. 2001, ApJ, 554, 190

de Blok, W.J.G., \& Walter, F. 2003, MNRAS 341, L39

Gerhard, O., \& Silk, J., 1996 ApJ, 472, 34

Gibson, S.J., Taylor, A.R., Higgs, L.A., \& Dewdney, P.E. 2000, ApJ, 540, 851

Heithausen, A., 2004, ApJ, 606, L13

Kalberla, P.M.W. 2003, ApJ, 588, 805

Larson, R. 1969, MNRAS, 145, 271

Masset, F. S. \& Bureau, M. 2003, ApJ, 586, 152

McGaugh, S. S., Schombert, J. M., Bothun, G. D., \& de Blok, W. J. G. 2000, ApJ, 533, L99

Pfenniger, D. \& Combes, F. 1994, A\&A, 285, 94

Pfenniger, D. \& Revaz, Y. 2005, A\&A, in press

Reach, W.T., Dwek, E., Fixsen, D.J., Hewagama, T., Mather, J.C., et al. 1995, ApJ, 451, 188

Revaz, Y., \& Pfenniger, D. 2004, A\&A, 425, 67

Wardle, M., \& Walker, M. 1999, ApJ, 527, L109

$087 / 4$ 